\title{
Existence and Iteration of Positive Solutions for One-Dimensional $p$-Laplacian Boundary Value Problems with Dependence on the First-Order Derivative
}

\author{
Zhiyong Wang and Jihui Zhang \\ Institute of Mathematics, School of Mathematics and Computer Sciences, Nanjing Normal University, \\ Jiangsu, Nanjing 210097, China
}

Correspondence should be addressed to Zhiyong Wang, mathswzhy@126.com

Received 7 April 2008; Accepted 18 May 2008

Recommended by Zhitao Zhang

This paper deals with the existence and iteration of positive solutions for the following onedimensional $p$-Laplacian boundary value problems: $\left(\phi_{p}\left(u^{\prime}(t)\right)\right)^{\prime}+a(t) f\left(t, u(t), u^{\prime}(t)\right)=0, t \in(0,1)$, subject to some boundary conditions. By making use of monotone iterative technique, not only we obtain the existence of positive solutions for the problems, but also we establish iterative schemes for approximating the solutions.

Copyright (C) 2008 Z. Wang and J. Zhang. This is an open access article distributed under the Creative Commons Attribution License, which permits unrestricted use, distribution, and reproduction in any medium, provided the original work is properly cited.

\section{Introduction}

In this paper, we are concerned with the existence and iteration of positive solutions for the following one-dimensional $p$-Laplacian boundary value problems:

$$
\left(\phi_{p}\left(u^{\prime}(t)\right)\right)^{\prime}+a(t) f\left(t, u(t), u^{\prime}(t)\right)=0, \quad t \in(0,1),
$$

subject to one of the following boundary conditions:

$$
u^{\prime}(0)=0, \quad \alpha u(1)+\beta u^{\prime}(1)=0,
$$

or

$$
\gamma u(0)-\delta u^{\prime}(0)=0, \quad u^{\prime}(1)=0,
$$

where $\phi_{p}(s)=|s|^{p-2} s$ with $p>1,\left(\phi_{p}\right)^{-1}=\phi_{q}, 1 / p+1 / q=1, \alpha, \beta, \gamma, \delta>0$ and $f, a$ satisfy the following: 
$\left(\mathrm{H}_{1}\right) f:[0,1] \times[0,+\infty) \times(-\infty, 0] \rightarrow[0,+\infty)$ is continuous;

$\left(\mathrm{H}_{1}^{*}\right) f:[0,1] \times[0,+\infty) \times[0,+\infty) \rightarrow[0,+\infty)$ is continuous;

$\left(\mathrm{H}_{2}\right) f(t, u, v)$ is nondecreasing in $u-v$ for all $t \in[0,1]$, that is, $f(t, u, v) \geq f(t, x, y)$ for all $u-v \geq x-y, t \in[0,1]$

$\left(\mathrm{H}_{2}^{*}\right) f(t, u, v)$ is nondecreasing in $u+v$ for all $t \in[0,1]$, that is, $f(t, u, v) \geq f(t, x, y)$ for all $u+v \geq x+y, t \in[0,1]$

$\left(\mathrm{H}_{3}\right) a:(0,1) \rightarrow[0,+\infty)$ is measurable, and $a(t)$ is not identically zero on any compact subinterval of $(0,1)$. Furthermore, $a(t)$ satisfies $0<\int_{0}^{1} a(t) d t<+\infty$.

Here, a positive solution of (1.1), (1.2) or (1.1), (1.3) means a solution $u^{*}(t)$ of $(1.1),(1.2)$ or (1.1), (1.3) satisfying $u^{*}(t)>0,0<t<1$.

The boundary value problems (1.1), (1.2) and (1.1), (1.3) deserve a special mention because these forms occur in the study of the $n$-dimensional $p$-Laplacian equation, nonNewtonian fluid theory and turbulent flow of a gas in a porous medium [1].

A consistent account on the existing literature on equation

$$
\left(\phi_{p}\left(u^{\prime}(t)\right)\right)^{\prime}+a(t) f(t, u(t))=0, \quad t \in(0,1),
$$

is provided and it emphasizes the use of upper and lower solution technique and the fixed point theory, for instance, Krasnoselskii fixed point theorem, the fixed point index of a completely continuous operator with respect to a cone in a Banach space, one may see [25] and the references therein. In [6], by using the monotone iterative technique, Ma et al. obtained the existence of monotone positive solution and established the corresponding iterative schemes of (1.4) under the multipoint boundary value condition. However, in their discussion, the nonlinear term $f$ is not involved with the first-order derivative $u^{\prime}(t)$.

Recently, there is much attention focused on the study of the boundary value problems like (1.1) which the nonlinear term $f$ is involved with the first-order derivative explicitly. In [7], Bai et al. considered the boundary value problems (1.1), (1.2) and (1.1), (1.3) and they proved that problems (1.1), (1.2) and (1.1), (1.3) possessed at least three positive solutions by applying a fixed point theorem due to Avery and Peterson [8]. In [9], the authors also deal with the boundary value problem (1.1), (1.2) via Krasnoselskii fixed point theorem. Here, we should mention that Sun and Ge [10] have got the positive solution of the boundary value problem (1.1), (1.2) by making use of monotone iterative technique.

On the other hand, when $f$ is involved with the first-order derivative explicitly, we can see easily that the results obtained in $[1,7,9]$ are only the existence of positive solutions under some suitable conditions. Seeing such a fact, it is an interesting problem which shows how to find these solutions since they exist definitely. Motivated by the above-mentioned results, by making use of the classical monotone iterative technique, we will investigate not only the existence of positive solutions for the boundary value problems (1.1), (1.2) and (1.1), (1.3), but also give iterative schemes for approximating the solutions. Unlike the idea of [10], we will construct a special subset $K$ (see Section 3) and look at $u(t)-u^{\prime}(t)$ or $u(t)+u^{\prime}(t)$ as a unit to overcome difficulties when $f$ depends on both $u(t)$ and $u^{\prime}(t)$. It is worth starting that the first term of our iterative schemes are simple functions which are determined with some linear ordinary equations and cone $P$ (see Remark 3.2). Therefore, the iterative schemes are significant and feasible. At the same time, we will correct some mistakes in [11-13] (see Remark 3.5). 
This paper is organized as follows. After this section, some definitions and lemmas will be established in Section 2. In Section 3, we will give our main results Theorems 3.1 and 3.4. Finally, an example is also presented to illustrate our results in Section 4.

\section{Preliminaries}

In this section, we provide some background material from the theory of cones in Banach spaces. We also state some lemmas which are important to proof our main results.

Definition 2.1. Let $X$ be a real Banach space. A nonempty closed set $P \subset X$ is called a cone, if it satisfies the following two conditions:

(i) $a_{1} u+a_{2} v \in P$, for all $u, v \in P$ and all $a_{1} \geq 0, a_{2} \geq 0$;

(ii) $u \in P,-u \in P$ implies $u=0$.

Definition 2.2. A map $\tau$ is said to be concave on $[0,1]$, if

$$
\tau(t u+(1-t) v) \geq t \tau(u)+(1-t) \tau(v),
$$

for all $u, v \in[0,1]$ and $t \in[0,1]$.

Consider the Banach space $X:=C^{1}[0,1]$ equipped with the norm

$$
\|u\|:=\max _{0 \leq t \leq 1}\left[|u(t)|+\left|u^{\prime}(t)\right|\right]
$$

and define the cone $P \subset X$ by

$$
P:=\{u(t) \in X: u(t) \geq 0, u(t) \text { is concave and nonincreasing on }[0,1]\} .
$$

Lemma 2.3 (see [3]). If $u(t) \in P$, then $u(t) \geq q(t) \max _{0 \leq t \leq 1}|u(t)|$, where $q(t):=1-t, t \in[0,1]$.

Define the operator $A: X \rightarrow X$ as follows:

$$
(A u)(t):=\frac{\beta}{\alpha} \phi_{q}\left(\int_{0}^{1} a(r) f\left(r, u(r), u^{\prime}(r)\right) d r\right)+\int_{t}^{1} \phi_{q}\left(\int_{0}^{s} a(r) f\left(r, u(r), u^{\prime}(r)\right) d r\right) d s, \quad t \in[0,1] .
$$

Lemma 2.4. Assume $\left(H_{1}\right),\left(H_{3}\right)$ hold, then $A: P \rightarrow P$ is completely continuous.

Proof. From $\left(\mathrm{H}_{1}\right),\left(\mathrm{H}_{3}\right)$, it is obviously that $(A u)(t) \geq 0$. Since $(A u)^{\prime}(t) \leq 0$, we can see that $(A u)^{\prime}(t)$ is continuous and nonincreasing on $[0,1]$, that is, $(A u)(t)$ is concave on $[0,1]$, so $A$ : $P \rightarrow P$ is well defined. The continuity of $A$ is clear because of the continuity of $f$ and $a$. Now, we will prove that $A$ is compact. Let $\Omega \subset P$ be a bounded set, then there exists $D$, such that $\Omega \subset\{u(t) \in P:\|u\| \leq D\}$. For any $u(t) \in \Omega$, we have $0 \leq \int_{0}^{1} a(r) f\left(r, u(r), u^{\prime}(r)\right) d r \leq$ $\max _{t \in[0,1], u \in[0, D], v \in[-D, 0]} f(t, u(t), v(t)) \int_{0}^{1} a(t) d t:=E$, then we have

$$
|(A u)(t)| \leq\left(\frac{\beta}{\alpha}+1\right) \phi_{q}(E), \quad\left|(A u)^{\prime}(t)\right| \leq \phi_{q}(E), \quad\left|\left(\phi_{p}\left((A u)^{\prime}(t)\right)\right)^{\prime}\right| \leq E .
$$

The Arzela-Ascoli theorem guarantees that $A \Omega$ is relatively compact, which means that $A$ is compact. Then $A: P \rightarrow P$ is completely continuous.

Lemma 2.5. Assume $\left(H_{1}\right),\left(H_{2}\right)$ and $\left(H_{3}\right)$ hold. If $\omega_{1}(t)$, $\omega_{2}(t) \in P$ such that $\omega_{1}(t)-\omega_{1}^{\prime}(t) \geq \omega_{2}(t)-$ $\omega_{2}^{\prime}(t), t \in[0,1]$, then $\left(A \omega_{1}\right)(t) \geq\left(A \omega_{2}\right)(t),\left(A \omega_{1}\right)^{\prime}(t) \leq\left(A \omega_{2}\right)^{\prime}(t), t \in[0,1]$. 
Proof. Noticing that $f(t, u, v)$ is nondecreasing in $u-v, t \in[0,1]$, the proof is simple, here we omit it.

\section{Main results}

For convenience, we denote

$$
\begin{array}{rlrl}
C_{1}:=\min & \left\{1, \frac{\beta}{\alpha}\right\}, & C_{2}:=1+\frac{\beta}{\alpha}, & S_{1}:=\left(1+C_{1}^{-1}\right)^{-1}, \quad S_{2}:=8 C_{1}^{-1} C_{2}, \\
C_{3}:=\min \left\{1, \frac{\delta}{\gamma}\right\}, & C_{4}:=1+\frac{\delta}{\gamma}, & S_{3}:=\left(1+C_{3}^{-1}\right)^{-1}, \quad S_{4}:=8 C_{3}^{-1} C_{4}, \\
M_{1}:=\left[\left(\frac{\beta}{\alpha}+2\right) \phi_{q}\left(\int_{0}^{1} a(r) d r\right)\right]^{-1}, & N_{1}:=\left[\frac{\beta}{\alpha} \phi_{q}\left(\int_{\theta_{1}}^{1-\theta_{1}} a(r) d r\right)\right]^{-1}, \\
M_{2}:=\left[\left(\frac{\delta}{\gamma}+2\right) \phi_{q}\left(\int_{0}^{1} a(r) d r\right)\right]^{-1}, & N_{2}:=\left[\frac{\delta}{\gamma} \phi_{q}\left(\int_{\theta_{2}}^{1-\theta_{2}} a(r) d r\right)\right]^{-1},
\end{array}
$$

where $\theta_{1} \in\left(0,\left(2 C_{2}\right)^{-1}\right) \subset(0,1 / 2), \theta_{2} \in\left(0,\left(2 C_{4}\right)^{-1}\right) \subset(0,1 / 2)$. It is easy to see that $0<S_{1}, S_{3}<$ $1, S_{2}, S_{4}>8$.

Theorem 3.1. Assume $\left(H_{1}\right),\left(H_{2}\right)$, and $\left(H_{3}\right)$ hold. Moreover, suppose that there exist six constants $R_{i}, r_{i}, L_{i}, i=1,2$, with $R_{1}, r_{1}, L_{1}>0, R_{2}, r_{2}, L_{2}<0, S_{1}\left(L_{1}-L_{2}\right)>S_{2}\left(r_{1}-r_{2}\right)$ and $R_{1}-R_{2}:=$ $4 \theta_{1} C_{2}\left(r_{1}-r_{2}\right)$, such that

$$
\begin{aligned}
& \left(\mathrm{H}_{4}\right) \max _{0 \leq t \leq 1} f\left(t, L_{1}, L_{2}\right) \leq \phi_{p}\left[\left(L_{1}-L_{2}\right) S_{1} M_{1}\right] \\
& \left(\mathrm{H}_{5}\right) \min _{\theta_{1} \leq t \leq 1-\theta_{1}} f\left(t, R_{1}, R_{2}\right) \geq \phi_{p}\left[\left(r_{1}-r_{2}\right) S_{2} N_{1}\right] .
\end{aligned}
$$

Then the boundary value problem (1.1), (1.2) has at least two nonincreasing positive solutions $u^{*}(t)$ and $v^{*}(t) \in P$ with

$$
S_{2}\left(r_{1}-r_{2}\right) \leq\left\|u^{*}\right\| \leq S_{1}\left(L_{1}-L_{2}\right), \quad S_{2}\left(r_{1}-r_{2}\right) \leq\left\|v^{*}\right\| \leq S_{1}\left(L_{1}-L_{2}\right),
$$

and $\lim _{n \rightarrow \infty}\left(A^{n} u_{0}\right)(t)=u^{*}(t), \lim _{n \rightarrow \infty}\left(A^{n} v_{0}\right)(t)=v^{*}(t)$, where

$$
u_{0}(t)=L_{1}-L_{2}+C_{5} e^{t}, \quad v_{0}(t)=(q(t)+1)\left(r_{1}-r_{2}\right)+C_{6} e^{t}, \quad t \in[0,1]
$$

$C_{5}$ and $C_{6}$ are arbitrary constants which satisfy $-e^{-1}\left(L_{1}-L_{2}\right) \leq C_{5} \leq 0,-e^{-1}\left(r_{1}-r_{2}\right) \leq C_{6} \leq 0$.

Proof. We denote a set $K \subset P$ by

$$
\begin{aligned}
K:=\left\{u(t) \in P: C_{1} \max _{0 \leq t \leq 1}\left|u^{\prime}(t)\right| \leq|u(t)|, \max _{0 \leq t \leq 1}|u(t)| \leq C_{2} \max _{0 \leq t \leq 1}\left|u^{\prime}(t)\right|,\right. \\
\left.S_{2}\left(r_{1}+\left|r_{2}\right|\right) \leq\|u\| \leq S_{1}\left(L_{1}+\left|L_{2}\right|\right)\right\} .
\end{aligned}
$$

Based on the preceding preliminaries, we can divide our proof into three steps. 
Step 1. We first prove $A(K) \subset K$. Let $u(t) \in K$, note that $u(t), u^{\prime}(t)$ is nonincreasing on $[0,1]$, then

$$
0 \leq u(t)-u^{\prime}(t) \leq \max _{0 \leq t \leq 1}\left[|u(t)|+\left|u^{\prime}(t)\right|\right]=\|u\| \leq S_{1}\left(L_{1}-L_{2}\right) \leq L_{1}-L_{2}, \quad t \in[0,1]
$$

By Lemma 2.3, we have

$$
\begin{aligned}
u(t)-u^{\prime}(t) & \geq \min _{\theta_{1} \leq t \leq 1-\theta_{1}}|u(t)| \\
& \geq \min _{\theta_{1} \leq t \leq 1-\theta_{1}} q(t) \max _{0 \leq t \leq 1}|u(t)| \\
& =\frac{1}{2} \theta_{1}\left[\max _{0 \leq t \leq 1}|u(t)|+\max _{0 \leq t \leq 1}|u(t)|\right] \\
& \geq \frac{1}{2} \theta_{1}\left[\max _{0 \leq t \leq 1}|u(t)|+C_{1} \max _{0 \leq t \leq 1}\left|u^{\prime}(t)\right|\right] \\
& \geq \frac{1}{2} \theta_{1} \min \left\{1, C_{1}\right\}\|u\| \\
& \geq \frac{1}{2} \theta_{1} C_{1} S_{2}\left(r_{1}-r_{2}\right) \\
& =4 \theta_{1} C_{2}\left(r_{1}-r_{2}\right) \\
& =R_{1}-R_{2}, \quad t \in\left[\theta_{1}, 1-\theta_{1}\right] .
\end{aligned}
$$

Since $f(t, u, v)$ is nondecreasing in $u-v, t \in[0,1]$, and by assumptions $\left(\mathrm{H}_{4}\right)$ and $\left(\mathrm{H}_{5}\right)$, we obtain

$$
\begin{aligned}
& 0 \leq f\left(t, u(t), u^{\prime}(t)\right) \leq f\left(t, L_{1}, L_{2}\right) \leq \phi_{p}\left[\left(L_{1}-L_{2}\right) S_{1} M_{1}\right], \quad t \in[0,1], \\
& f\left(t, u(t), u^{\prime}(t)\right) \geq f\left(t, R_{1}, R_{2}\right) \geq \phi_{p}\left[\left(r_{1}-r_{2}\right) S_{2} N_{1}\right], \quad t \in\left[\theta_{1}, 1-\theta_{1}\right]
\end{aligned}
$$


which imply that

$$
\begin{aligned}
\|A u\|= & \max _{0 \leq t \leq 1}\left[|(A u)(t)|+\left|(A u)^{\prime}(t)\right|\right] \\
= & \max _{0 \leq t \leq 1}\left\{\phi_{q}\left(\int_{0}^{t} a(r) f\left(r, u(r), u^{\prime}(r)\right) d r\right)+\frac{\beta}{\alpha} \phi_{q}\left(\int_{0}^{1} a(r) f\left(r, u(r), u^{\prime}(r)\right) d r\right)\right. \\
& \left.\quad+\int_{t}^{1} \phi_{q}\left(\int_{0}^{s} a(r) f\left(r, u(r), u^{\prime}(r)\right) d r\right) d s\right\} \\
\leq & \left(\frac{\beta}{\alpha}+2\right) \phi_{q}\left(\int_{0}^{1} a(r) f\left(r, u(r), u^{\prime}(r)\right) d r\right) \\
\leq & \left(\frac{\beta}{\alpha}+2\right) \phi_{q}\left(\int_{0}^{1} a(r) f\left(r, L_{1}, L_{2}\right) d r\right) \\
\leq & \left(\frac{\beta}{\alpha}+2\right) \phi_{q}\left(\int_{0}^{1} a(r) d r\right) S_{1}\left(L_{1}-L_{2}\right) M_{1} \\
= & S_{1}\left(L_{1}-L_{2}\right),
\end{aligned}
$$

$$
\begin{aligned}
\|A u\| & =\max _{0 \leq t \leq 1}\left[|(A u)(t)|+\left|(A u)^{\prime}(t)\right|\right] \\
& \geq \max _{0 \leq t \leq 1}|(A u)(t)| \\
& \geq \frac{\beta}{\alpha} \phi_{q}\left(\int_{0}^{1} a(r) f\left(r, u(r), u^{\prime}(r)\right) d r\right) \\
& \geq \frac{\beta}{\alpha} \phi_{q}\left(\int_{\theta_{1}}^{1-\theta_{1}} a(r) f\left(r, R_{1}, R_{2}\right) d r\right) \\
& \geq \frac{\beta}{\alpha} \phi_{q}\left(\int_{\theta_{1}}^{1-\theta_{1}} a(r) d r\right) S_{2}\left(r_{1}-r_{2}\right) N_{1} \\
& =S_{2}\left(r_{1}-r_{2}\right) .
\end{aligned}
$$


On the other hand, for $u(t) \in K$, we have

$$
\begin{aligned}
|(A u)(t)|= & \frac{\beta}{\alpha} \phi_{q}\left(\int_{0}^{1} a(r) f\left(r, u(r), u^{\prime}(r)\right) d r\right) \\
& +\int_{t}^{1} \phi_{q}\left(\int_{0}^{s} a(r) f\left(r, u(r), u^{\prime}(r)\right) d r\right) d s \\
\geq & \frac{\beta}{\alpha} \phi_{q}\left(\int_{0}^{1} a(r) f\left(r, u(r), u^{\prime}(r)\right) d r\right) \\
\geq & C_{1} \max _{0 \leq t \leq 1}(A u)^{\prime}(t) \mid, \\
\max _{0 \leq t \leq 1}|(A u)(t)|= & \frac{\beta}{\alpha} \phi_{q}\left(\int_{0}^{1} a(r) f\left(r, u(r), u^{\prime}(r)\right) d r\right) \\
& +\int_{0}^{1} \phi_{q}\left(\int_{0}^{s} a(r) f\left(r, u(r), u^{\prime}(r)\right) d r\right) d s \\
\leq & \left(\frac{\beta}{\alpha}+1\right) \phi_{q}\left(\int_{0}^{1} a(r) f\left(r, u(r), u^{\prime}(r)\right) d r\right) \\
= & C_{2} \max _{0 \leq t \leq 1}\left|(A u)^{\prime}(t)\right| .
\end{aligned}
$$

In virtue of (3.8)-(3.9), $A(K) \subset K$.

Step 2. Let $u_{0}(t)=L_{1}-L_{2}+C_{5} e^{t}, C_{5}$ is an arbitrary constant which satisfies $-e^{-1}\left(L_{1}-L_{2}\right) \leq$ $C_{5} \leq 0, t \in[0,1]$, then $u_{0}(t) \geq 0, u_{0}^{\prime}(t)=C_{5} e^{t} \leq 0, u_{0}^{\prime \prime}(t)=C_{5} e^{t} \leq 0, t \in[0,1]$. Hence, $u_{0}(t) \in P, u_{0}(t)-u_{0}^{\prime}(t)=L_{1}-L_{2}, t \in[0,1]$. Let $u_{1}(t)=\left(A u_{0}\right)(t)$, next we claim that $u_{1}(t) \in K$. Indeed, it is easy to check that

$$
\begin{gathered}
\left|u_{1}(t)\right|=\left|\left(A u_{0}\right)(t)\right| \geq C_{1} \max _{0 \leq t \leq 1}\left|\left(A u_{0}\right)^{\prime}(t)\right|=C_{1} \max _{0 \leq t \leq 1}\left|u_{1}^{\prime}(t)\right|, \\
\max _{0 \leq t \leq 1}\left|u_{1}(t)\right|=\max _{0 \leq t \leq 1}\left|\left(A u_{0}\right)^{\prime}(t)\right| \leq C_{2} \max _{0 \leq t \leq 1}\left|\left(A u_{0}\right)^{\prime}(t)\right|=C_{2} \max _{0 \leq t \leq 1}\left|u_{1}^{\prime}(t)\right| .
\end{gathered}
$$


Using assumptions $S_{1}\left(L_{1}-L_{2}\right)>S_{2}\left(r_{1}-r_{2}\right)$ and $R_{1}-R_{2}=4 \theta_{1} C_{2}\left(r_{1}-r_{2}\right)$, we have $u_{0}(t)-u_{0}^{\prime}(t)=$ $L_{1}-L_{2} \geq 8\left(r_{1}-r_{2}\right) \geq 4 \theta_{1} C_{2}\left(r_{1}-r_{2}\right)=R_{1}-R_{2}, t \in\left[\theta_{1}, 1-\theta_{1}\right]$, these imply that

$$
\begin{aligned}
\left\|u_{1}\right\|=\left\|A u_{0}\right\| & =\max _{0 \leq t \leq 1}\left[\left|\left(A u_{0}\right)(t)\right|+\left|\left(A u_{0}\right)^{\prime}(t)\right|\right] \\
& \leq\left(\frac{\beta}{\alpha}+2\right) \phi_{q}\left(\int_{0}^{1} a(r) f\left(r, u_{0}(r), u_{0}^{\prime}(r)\right) d r\right) \\
& \leq\left(\frac{\beta}{\alpha}+2\right) \phi_{q}\left(\int_{0}^{1} a(r) f\left(r, L_{1}, L_{2}\right) d r\right) \\
& \leq\left(\frac{\beta}{\alpha}+2\right) \phi_{q}\left(\int_{0}^{1} a(r) d r\right) S_{1}\left(L_{1}-L_{2}\right) M_{1} \\
& \leq S_{1}\left(L_{1}-L_{2}\right), \\
\left\|u_{1}\right\|=\left\|A u_{0}\right\| & =\max _{0 \leq t \leq 1}\left[\left|\left(A u_{0}\right)(t)\right|+\left|\left(A u_{0}\right)^{\prime}(t)\right|\right] \\
& \geq \frac{\beta}{\alpha} \phi_{q}\left(\int_{\theta_{1}}^{1-\theta_{1}} a(r) f\left(r, u_{0}(r), u_{0}^{\prime}(r)\right) d r\right) \\
& \geq \frac{\beta}{\alpha} \phi_{q}\left(\int_{\theta_{1}}^{1-\theta_{1}} a(r) f\left(r, R_{1}, R_{2}\right) d r\right) \\
& \geq \frac{\beta}{\alpha} \phi_{q}\left(\int_{\theta_{1}}^{1-\theta_{1}} a(r) d r\right) S_{2}\left(r_{1}-r_{2}\right) N_{1} \\
& =S_{2}\left(r_{1}-r_{2}\right) .
\end{aligned}
$$

Therefore, $u_{1}(t) \in K$. We denote

$$
u_{n+1}(t):=\left(A u_{n}\right)(t)=\left(A^{n+1} u_{0}\right)(t), \quad n=0,1,2, \ldots
$$

Since $A(K) \subset K, u_{n}(t) \in K, n=1,2, \ldots$. From Lemma 2.4, $A$ is compact, we assert that $\left\{u_{n}\right\}_{n=1}^{\infty}$ has a convergent subsequence $\left\{u_{n_{k}}\right\}_{k=1}^{\infty}$ and there exists $u^{*}(t) \in K$, such that $u_{n_{k}}(t) \rightarrow u^{*}(t)$. Now, since $u_{1}(t) \in K \subset P$, for $t \in[0,1]$, we have

$$
\begin{aligned}
u_{1}(t)-u_{1}^{\prime}(t) & \leq\left(1+C_{1}^{-1}\right)\left|u_{1}(t)\right| \leq\left(1+C_{1}^{-1}\right)\left\|u_{1}\right\| \\
& \leq\left(1+C_{1}^{-1}\right) S_{1}\left(L_{1}-L_{2}\right)=L_{1}-L_{2}=u_{0}(t)-u_{0}^{\prime}(t) .
\end{aligned}
$$

This combined with Lemma 2.5 gives

$$
u_{2}(t)=\left(A u_{1}\right)(t) \leq\left(A u_{0}\right)(t)=u_{1}(t), \quad u_{2}^{\prime}(t)=\left(A u_{1}\right)^{\prime}(t) \geq\left(A u_{0}\right)^{\prime}(t)=u_{1}^{\prime}(t),
$$

so

$$
u_{2}(t)-u_{2}^{\prime}(t) \leq u_{1}(t)-u_{1}^{\prime}(t), \quad 0 \leq t \leq 1
$$


By induction,

$$
u_{n+1}(t) \leq u_{n}(t), \quad u_{n+1}^{\prime}(t) \geq u_{n}^{\prime}(t), \quad 0 \leq t \leq 1, n=1,2, \ldots
$$

Hence, we assert that $u_{n}(t) \rightarrow u^{*}(t)$. Let $n \rightarrow \infty$ in (3.12) to obtain $\left(A u^{*}\right)(t)=u^{*}(t)$ since $A$ is continuous. Since $\left\|u^{*}\right\| \geq S_{2}\left(r_{1}-r_{2}\right)>0$ and $u^{*}(t)$ is a nonnegative concave function on $[0,1]$, we conclude that $u^{*}(t)>0, t \in(0,1)$. It is well known that the fixed point of operator $A$ is the solution of the boundary value problem (1.1), (1.2). Therefore, $u^{*}(t)$ is a positive, nonincreasing solution of the boundary value problem (1.1), (1.2).

Step 3. Put $v_{0}(t)=(q(t)+1)\left(r_{1}-r_{2}\right)+C_{6} e^{t}, C_{6}$ is an arbitrary constant satisfying $-e^{-1}\left(r_{1}-r_{2}\right) \leq$ $C_{6} \leq 0, t \in[0,1]$, then $v_{0}(t) \geq 0, v_{0}^{\prime}(t)=-\left(r_{1}-r_{2}\right)+C_{6} e^{t} \leq 0, v_{0}^{\prime \prime}(t)=C_{6} e^{t} \leq 0, t \in[0,1]$. Hence, $v_{0}(t) \in P$. From the definition of $\theta_{1}$ and $R_{1}-R_{2}$, we derive that

$$
\begin{aligned}
v_{0}(t)-v_{0}^{\prime}(t) & =(q(t)+2)\left(r_{1}-r_{2}\right) \geq\left(2+\theta_{1}\right)\left(r_{1}-r_{2}\right) \\
& \geq 2\left(r_{1}-r_{2}\right) \geq 4 \theta_{1} C_{2}\left(r_{1}-r_{2}\right)=R_{1}-R_{2}, \quad t \in\left[\theta_{1}, 1-\theta_{1}\right], \\
v_{0}(t)-v_{0}^{\prime}(t) & =(q(t)+2)\left(r_{1}-r_{2}\right) \leq 3\left(r_{1}-r_{2}\right) \leq L_{1}-L_{2}, \quad t \in[0,1] .
\end{aligned}
$$

Setting $v_{1}(t)=\left(A v_{0}\right)(t)$, in what follows, we will prove that $v_{1}(t) \in K$. In fact, similar to (3.10)(3.11), combined with the above inequalities, one has

$$
\begin{aligned}
\left|v_{1}(t)\right| & =\left|\left(A v_{0}\right)(t)\right| \geq C_{1} \max _{0 \leq t \leq 1}\left|\left(A v_{0}\right)^{\prime}(t)\right|=C_{1} \max _{0 \leq t \leq 1}\left|v_{1}^{\prime}(t)\right| \\
\max _{0 \leq t \leq 1}\left|v_{1}(t)\right| & =\max _{0 \leq t \leq 1}\left|\left(A v_{0}\right)^{\prime}(t)\right| \leq C_{2} \max _{0 \leq t \leq 1}\left|\left(A v_{0}\right)^{\prime}(t)\right|=C_{2} \max _{0 \leq t \leq 1}\left|u_{1}^{\prime}(t)\right|, \\
\left\|v_{1}\right\|=\left\|A v_{0}\right\| & =\max _{0 \leq t \leq 1}\left[\left|\left(A v_{0}\right)(t)\right|+\left|\left(A v_{0}\right)^{\prime}(t)\right|\right] \\
& \leq\left(\frac{\beta}{\alpha}+2\right) \phi_{q}\left(\int_{0}^{1} a(r) f\left(r, v_{0}(r), v_{0}^{\prime}(r)\right) d r\right) \\
& \leq\left(\frac{\beta}{\alpha}+2\right) \phi_{q}\left(\int_{0}^{1} a(r) f\left(r, L_{1}, L_{2}\right) d r\right) \\
& \leq S_{1}\left(L_{1}-L_{2}\right) \\
\left\|v_{1}\right\|=\left\|A v_{0}\right\| & =\max _{0 \leq t \leq 1}\left[\left|\left(A v_{0}\right)(t)\right|+\left|\left(A v_{0}\right)^{\prime}(t)\right|\right] \\
& \geq \frac{\beta}{\alpha} \phi_{q}\left(\int_{\theta_{1}}^{1-\theta_{1}} a(r) f\left(r, v_{0}(r), v_{0}^{\prime}(r)\right) d r\right) \\
& \geq \frac{\beta}{\alpha} \phi_{q}\left(\int_{\theta_{1}}^{1-\theta_{1}} a(r) f\left(r, R_{1}, R_{2}\right) d r\right) \\
& =S_{2}\left(r_{1}-r_{2}\right) .
\end{aligned}
$$

We deduce from (3.18) that $v_{1}(t) \in K$. Denote

$$
v_{n+1}(t):=\left(A v_{n}\right)(t)=\left(A^{n+1} v_{0}\right)(t), \quad n=0,1,2, \ldots
$$


Since $v_{1}(t) \in K \subset P$, from Lemma 2.4, we have

$$
\begin{aligned}
v_{1}(t)-v_{1}^{\prime}(t) \geq & \frac{1}{2}\left|v_{1}(t)\right|+\frac{1}{2}\left|v_{1}(t)\right| \\
\geq & \frac{1}{2} q(t) \max _{0 \leq t \leq 1}\left|v_{1}(t)\right|+\frac{1}{2} C_{1} \max _{0 \leq t \leq 1}\left|v_{1}^{\prime}(t)\right| \\
\geq & \frac{1}{4} q(t)\left[\max _{0 \leq t \leq 1}\left|v_{1}(t)\right|+C_{1} \max _{0 \leq t \leq 1}\left|v_{1}^{\prime}(t)\right|\right] \\
& +\frac{1}{4} C_{1}\left[C_{2}^{-1} \max _{0 \leq t \leq 1}\left|v_{1}(t)\right|+\max _{0 \leq t \leq 1}\left|v_{1}^{\prime}(t)\right|\right] \\
\geq & \frac{1}{4} C_{1} q(t)\left\|v_{1}\right\|+\frac{1}{4} C_{1} C_{2}^{-1}\left\|v_{1}\right\| \\
\geq & \frac{1}{4} C_{1} q(t) S_{2}\left(r_{1}-r_{2}\right)+\frac{1}{4} C_{1} C_{2}^{-1} S_{2}\left(r_{1}-r_{2}\right) \\
\geq & (q(t)+2)\left(r_{1}-r_{2}\right) \\
= & v_{0}(t)-v_{0}^{\prime}(t), \quad t \in[0,1] .
\end{aligned}
$$

By Lemma 2.5, we get

$$
v_{2}(t)=\left(A v_{1}\right)(t) \geq\left(A v_{0}\right)(t)=v_{1}(t), \quad v_{2}^{\prime}(t)=\left(A v_{1}\right)^{\prime}(t) \leq\left(A v_{0}\right)^{\prime}(t)=v_{1}^{\prime}(t)
$$

so $v_{2}(t)-v_{2}^{\prime}(t) \geq v_{1}(t)-v_{1}^{\prime}(t), 0 \leq t \leq 1$. By induction, $v_{n+1}(t) \geq v_{n}(t), v_{n+1}^{\prime}(t) \leq v_{n}^{\prime}(t), 0 \leq t \leq$ $1, n=1,2, \ldots$. Hence, we assert that $v_{n}(t) \rightarrow v^{*}(t)$, and $v^{*}(t)>0, t \in(0,1)$. Therefore, $v^{*}(t)$ is a positive, nonincreasing solution of the boundary value problem (1.1), (1.2).

Remark 3.2. (i) We can easily get that $u^{*}(t)$ and $v^{*}(t)$ are the maximal and minimal solutions of the boundary value problem (1.1), (1.2) in $K$. Of course $u^{*}$ and $v^{*}$ may coincide and then the boundary value problem (1.1), (1.2) has only one solution in $K$.

(ii) It is worth pointing out that $u_{0}(t), v_{0}(t) \notin K$. In fact, $u_{0}(t) \in G_{1}, v_{0}(t) \in G_{2}$, which are determined with some linear ordinary equations and the cone $P$, this is different from the results in $[6,10]$, where

$$
\begin{aligned}
& G_{1}:=\left\{u_{0}(t) \in P: u_{0}(t)-u_{0}^{\prime}(t)=L_{1}-L_{2}\right\} \\
& G_{2}:=\left\{v_{0}(t) \in P: v_{0}(t)-v_{0}^{\prime}(t)=(q(t)+2)\left(r_{1}-r_{2}\right)\right\} .
\end{aligned}
$$

Corollary 3.3. Assume $\left(H_{1}\right),\left(H_{2}\right)$ and $\left(H_{3}\right)$ hold, suppose that

$\left(\mathrm{H}_{6}\right) f_{0}:=\lim \sup _{|u(t)|+|v(t)| \rightarrow 0} \min _{\theta_{1} \leq t \leq 1-\theta_{1}}\left(f(t, u(t), v(t)) /(|u(t)|+|v(t)|)^{p-1}\right)>\phi_{p}\left(2 N_{1}\right.$ $\left./ \theta_{1} C_{1}\right)$

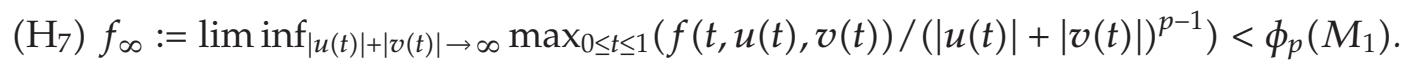


(Particularly, $f_{0}=+\infty, f_{\infty}=0$ ). Then there exist four constants $r_{i}, L_{i}, i=1,2$, with $r_{1}, L_{1}>$ $0, r_{2}, L_{2}<0, S_{1}\left(L_{1}-L_{2}\right)>S_{2}\left(r_{1}-r_{2}\right)$, such that the boundary value problem (1.1), (1.2) has at least two nonincreasing positive solutions $u^{*}(t)$ and $v^{*}(t) \in P$ with

$$
S_{2}\left(r_{1}-r_{2}\right) \leq\left\|u^{*}\right\| \leq S_{1}\left(L_{1}-L_{2}\right), \quad S_{2}\left(r_{1}-r_{2}\right) \leq\left\|v^{*}\right\| \leq S_{1}\left(L_{1}-L_{2}\right),
$$

and $\lim _{n \rightarrow \infty}\left(A^{n} u_{0}\right)(t)=u^{*}(t), \lim _{n \rightarrow \infty}\left(A^{n} v_{0}\right)(t)=v^{*}(t)$, where

$$
u_{0}(t)=L_{1}-L_{2}+C_{5} e^{t}, \quad v_{0}(t)=(q(t)+1)\left(r_{1}-r_{2}\right)+C_{6} e^{t}, \quad t \in[0,1]
$$

$C_{5}$ and $C_{6}$ are arbitrary constants which satisfy $-e^{-1}\left(L_{1}-L_{2}\right) \leq C_{5} \leq 0,-e^{-1}\left(r_{1}-r_{2}\right) \leq C_{6} \leq 0$.

Proof. It is very easy to verify the conditions $\left(\mathrm{H}_{4}\right)$ and $\left(\mathrm{H}_{5}\right)$ can be obtained from $\left(\mathrm{H}_{6}\right)$ and $\left(\mathrm{H}_{7}\right)$, so we omit the proof. theorem.

Obviously, though the similar arguments of Theorem 3.1, we could get the following

Theorem 3.4. Assume $\left(H_{1}^{*}\right),\left(H_{2}^{*}\right)$ and $\left(H_{3}^{*}\right)$ hold, suppose that there exist six positive constants $R_{j}, r_{j}, L_{j}, j=3,4$, with $S_{3}\left(L_{3}+L_{4}\right)>S_{4}\left(r_{3}+r_{4}\right)$ and $R_{3}+R_{4}:=4 \theta_{2} C_{4}\left(r_{3}+r_{4}\right)$, such that

$$
\begin{aligned}
& \left(\mathrm{H}_{8}\right) \max _{0 \leq t \leq 1} f\left(t, L_{3}, L_{4}\right) \leq \phi_{p}\left[\left(L_{3}+L_{4}\right) S_{3} M_{2}\right] \\
& \left(\mathrm{H}_{9}\right) \min _{\theta_{2} \leq t \leq 1-\theta_{2}} f\left(t, R_{3}, R_{4}\right) \geq \phi_{p}\left[\left(r_{3}+r_{4}\right) S_{4} N_{2}\right] .
\end{aligned}
$$

Then the boundary value problem (1.1), (1.3) has at least two nondecreasing positive solutions $\bar{u}^{*}(t)$ and $\bar{v}^{*}(t) \in \bar{P}$ with

$$
S_{4}\left(r_{3}+r_{4}\right) \leq\left\|\bar{u}^{*}\right\| \leq S_{3}\left(L_{3}+L_{4}\right), \quad S_{4}\left(r_{3}+r_{4}\right) \leq\left\|\bar{v}^{*}\right\| \leq S_{3}\left(L_{3}+L_{4}\right),
$$

and $\lim _{n \rightarrow \infty}\left(\bar{A}^{n} \bar{u}_{0}\right)(t)=\bar{u}^{*}(t), \lim _{n \rightarrow \infty}\left(\bar{A}^{n} \bar{v}_{0}\right)(t)=\bar{v}^{*}(t)$, where

$$
\bar{P}:=\{u(t) \in X: u(t) \geq 0, u(t) \text { is concave and nondecreasing on }[0,1]\} \text {. }
$$

$$
\begin{gathered}
(\bar{A} u)(t):=\frac{\delta}{r} \phi_{q}\left(\int_{0}^{1} a(r) f\left(r, u(r), u^{\prime}(r)\right) d r\right)+\int_{0}^{t} \phi_{q}\left(\int_{s}^{1} a(r) f\left(r, u(r), u^{\prime}(r)\right) d r\right) d s, \quad t \in[0,1], \\
\bar{u}_{0}(t)=L_{3}+L_{4}+C_{7} e^{-t}, \quad \bar{v}_{0}(t)=(t+1)\left(r_{3}+r_{4}\right)+C_{8} e^{-t}, \quad t \in[0,1] .
\end{gathered}
$$

$C_{7}$ and $C_{8}$ are arbitrary constants which satisfy $-\left(L_{3}+L_{4}\right) \leq C_{7} \leq 0,-\left(r_{3}+r_{4}\right) \leq C_{8} \leq 0$.

Remark 3.5. In [11], Liu and Zhang studied the following boundary value problem:

$$
\begin{aligned}
& \left(\phi\left(u^{\prime}(t)\right)\right)^{\prime}+a(t) f(t, u(t))=0, \quad t \in(0,1), \\
& u(0)-a_{1} u^{\prime}(0)=0, \quad u(1)+a_{2} u^{\prime}(1)=0,
\end{aligned}
$$


where $a_{1}, a_{2} \geq 0$ and $\phi$ is an increasing positive homomorphism and homeomorphism with $\phi(0)=0$ (for more details, see [11-13]). In that paper, the authors claimed that $u \in C[0,1] \cap$ $C^{1}(0,1)$ is a solution if and only if $u \in C[0,1]$ is a solution of the following integral equation:

$$
u(t)= \begin{cases}a_{1} \phi^{-1}\left(\int_{0}^{\tau} a(s) f(u(s)) d s\right)+\int_{0}^{t} \phi^{-1}\left(\int_{s}^{\tau} a(r) f(u(r)) d r\right) d s, & 0 \leq t \leq \tau, \\ a_{2} \phi^{-1}\left(\int_{\tau}^{1} a(s) f(u(s)) d s\right)+\int_{t}^{1} \phi^{-1}\left(\int_{\tau}^{s} a(r) f(u(r)) d r\right) d s, & \tau \leq t \leq 1,\end{cases}
$$

where $\tau=0$, if $u^{\prime}(0)=0 ; \tau=1$, if $u^{\prime}(1)=0$, otherwise $\tau$ is a solution of the equation

$$
g_{1}(t)=g_{2}(t),
$$

where

$$
\begin{aligned}
& g_{1}(t)=a_{1} \phi^{-1}\left(\int_{0}^{t} a(s) f(u(s)) d s\right)+\int_{0}^{t} \phi^{-1}\left(\int_{s}^{t} a(r) f(u(r)) d r\right) d s, \quad 0 \leq t<1, \\
& g_{2}(t)=a_{2} \phi^{-1}\left(\int_{t}^{1} a(s) f(u(s)) d s\right)+\int_{t}^{1} \phi^{-1}\left(\int_{t}^{s} a(r) f(u(r)) d r\right) d s, \quad 0<t \leq 1 .
\end{aligned}
$$

Unfortunately, such a claim is incorrect since

$$
\phi(-u) \neq-\phi(u)
$$

Similar reason, the results in $[12,13]$ are also incorrect, as they deal with the nonlinear systems. Under the boundary condition (1.3), we could avoid (3.31) to occur. With the similar argument of Theorem 3.4, we could have the similar theorem to Theorem 3.4 for the following boundary value problem:

$$
\begin{gathered}
\left(\phi\left(u^{\prime}(t)\right)\right)^{\prime}+a(t) f\left(t, u(t), u^{\prime}(t)\right)=0, \quad t \in(0,1), \\
\gamma u(0)-\delta u^{\prime}(0)=0, \quad u^{\prime}(1)=0 .
\end{gathered}
$$

Here, we omit the proofs.

\section{Example}

In this section, we will give an example to illustrate our results.

Example 4.1. Consider the boundary value problem

$$
\begin{gathered}
\left(\phi_{p}\left(u^{\prime}(t)\right)\right)^{\prime}+a(t) f\left(t, u(t), u^{\prime}(t)\right)=0, \quad t \in(0,1), \\
u^{\prime}(0)=0, \quad \alpha u(1)+\beta u^{\prime}(1)=0
\end{gathered}
$$

where $\phi_{p}(s)=|s|^{p-2} s$ with $p>1$, take $p=3, \alpha=1, \beta=1, a(t)=1, t \in[0,1]$,

$$
f(t, u, v)=t+u-v, \quad u \geq 0, v \leq 0, t \in[0,1],
$$

we have $q=3 / 2, C_{1}=1, C_{2}=2, S_{1}=1 / 2$ and $S_{2}=16$. Take $\theta_{1}=1 / 8$, we get $M_{1}=1 / 3, N_{1}=$ $2 \sqrt{3} / 3$. Choose $L_{1}=100, L_{2}=-100, r_{1}=0.01, r_{2}=-0.01, R_{1}=0.01$, and $R_{2}=-0.01$, so $f(t, u(t), v(t))$ satisfies the following: 
(1) $f:[0,1] \times[0,+\infty) \times(-\infty, 0] \rightarrow[0,+\infty)$ is continuous;

(2) $f(t, u, v)$ is nondecreasing in $u-v$ for all $t \in[0,1]$;

(3) $\max _{0 \leq t \leq 1} f\left(t, L_{1}, L_{2}\right)=\max _{0 \leq t \leq 1} f(t, 100,-100)=201 \leq \phi_{3}\left[\left(L_{1}-L_{2}\right) S_{1} M_{1}\right]=[(100+\mid-$ $100 \mid) \times 1 / 2 \times 1 / 3]^{2} \approx 1111.1 ;$

(4) $\min _{\theta_{1} \leq t \leq 1-\theta_{1}} f\left(t, R_{1}, R_{2}\right)=\min _{\theta_{1} \leq t \leq 1-\theta_{1}} f(t, 0.01,-0.01)=0.145 \geq \phi_{3}\left[\left(r_{1}-r_{2}\right) S_{2} N_{1}\right]=$ $[(0.01-0.01) \times 16 \times 2 \sqrt{3} / 3]^{2} \approx 0.1365$.

Therefore, by Theorem 3.1, the boundary value problem (4.1) has at least two nonincreasing positive solutions $u^{*}(t)$ and $v^{*}(t)$, such that

$$
0.32 \leq\left\|u^{*}\right\| \leq 100, \quad 0.32 \leq\left\|v^{*}\right\| \leq 100
$$

and $\lim _{n \rightarrow \infty}\left(A^{n} u_{0}\right)(t)=u^{*}(t), \lim _{n \rightarrow \infty}\left(A^{n} v_{0}\right)(t)=v^{*}(t)$, where

$$
u_{0}(t)=200+C_{5} e^{t}, \quad v_{0}(t)=0.02(2-t)+C_{6} e^{t}, \quad t \in[0,1]
$$

$C_{5}$ and $C_{6}$ are arbitrary constants which satisfy $-200 / e \leq C_{5} \leq 0,-0.02 / e \leq C_{6} \leq 0$.

For $n=1,2, \ldots$, the two iterative schemes are $u_{0}(t)=200+C_{5} e^{t}, t \in[0,1], C_{5}$ is arbitrary constant with $-200 / e \leq C_{5} \leq 0$,

$$
\begin{aligned}
u_{n+1}(t)=\left(A u_{n}\right)(t)= & {\left[\int_{0}^{1}\left(r+u_{n}(r)-u_{n}^{\prime}(r)\right) d r\right]^{1 / 2} } \\
& +\int_{t}^{1}\left(\int_{0}^{s}\left(r+u_{n}(r)-u_{n}^{\prime}(r)\right) d r\right)^{1 / 2} d s, \quad t \in[0,1]
\end{aligned}
$$

$v_{0}(t)=0.02(2-t)+C_{6} e^{t}, t \in[0,1], C_{6}$ is arbitrary constant with $-0.02 / e \leq C_{6} \leq 0$,

$$
\begin{aligned}
v_{n+1}(t)=\left(A v_{n}\right)(t)= & {\left[\int_{0}^{1}\left(r+v_{n}(r)-v_{n}^{\prime}(r)\right) d r\right]^{1 / 2} } \\
& +\int_{t}^{1}\left(\int_{0}^{s}\left(r+v_{n}(r)-v_{n}^{\prime}(r)\right) d r\right)^{1 / 2} d s, \quad t \in[0,1] .
\end{aligned}
$$

Remark 4.2. The nonlinear term $f(t, u, v)$ in $v$ is nonincreasing, so the results in [10] do not hold.

\section{Acknowledgments}

The project is supported by Foundation of Major Project of Science and Technology of Chinese Education Ministry, SRFDP of Higher Education, NSF of Education Committee of Jiangsu Province, and Graduate Innovation Foundation of Jiangsu Province (1612005022). 


\section{References}

[1] D. O'Regan, "Some general existence principles and results for $\left(\phi_{p}\left(y^{\prime}\right)\right)^{\prime}=q(t) f\left(t, y, y^{\prime}\right), 0<t<1$," SIAM Journal on Mathematical Analysis, vol. 24, no. 3, pp. 648-668, 1993.

[2] D. Jiang and W. Gao, "Upper and lower solution method and a singular boundary value problem for the one-dimensional p-Laplacian," Journal of Mathematical Analysis and Applications, vol. 252, no. 2, pp. 631-648, 2000.

[3] J. Wang, "The existence of positive solutions for the one-dimensional p-Laplacian," Proceedings of the American Mathematical Society, vol. 125, no. 8, pp. 2275-2283, 1997.

[4] Y. Guo and W. Ge, "Three positive solutions for the one-dimensional p-Laplacian," Journal of Mathematical Analysis and Applications, vol. 286, no. 2, pp. 491-508, 2003.

[5] L. Kong and J. Wang, "Multiple positive solutions for the one-dimensional p-Laplacian," Nonlinear Analysis: Theory, Methods \& Applications, vol. 42, no. 8, pp. 1327-1333, 2000.

[6] D.-X. Ma, Z.-J. Du, and W.-G. Ge, "Existence and iteration of monotone positive solutions for multipoint boundary value problem with $p$-Laplacian operator," Computers \& Mathematics with Applications, vol. 50, no. 5-6, pp. 729-739, 2005.

[7] Z. Bai, Z. Gui, and W. Ge, "Multiple positive solutions for some $p$-Laplacian boundary value problems," Journal of Mathematical Analysis and Applications, vol. 300, no. 2, pp. 477-490, 2004.

[8] R. I. Avery and A. C. Peterson, "Three positive fixed points of nonlinear operators on ordered Banach spaces," Computers \& Mathematics with Applications, vol. 42, no. 3-5, pp. 313-322, 2001.

[9] Z. Wang and J. Zhang, "Positive solutions for one-dimensional $p$-Laplacian boundary value problems with dependence on the first order derivative," Journal of Mathematical Analysis and Applications, vol. 314, no. 2, pp. 618-630, 2006.

[10] B. Sun and W. Ge, "Existence and iteration of positive solutions for some $p$-Laplacian boundary value problems," Nonlinear Analysis: Theory, Methods \& Applications, vol. 67, no. 6, pp. 1820-1830, 2007.

[11] B. Liu and J. Zhang, "The existence of positive solutions for some nonlinear boundary value problems with linear mixed boundary conditions," Journal of Mathematical Analysis and Applications, vol. 309, no. 2, pp. 505-516, 2005.

[12] B. Liu and J. Zhang, "The existence of positive solutions for some nonlinear equation systems," Journal of Mathematical Analysis and Applications, vol. 324, no. 2, pp. 970-981, 2006.

[13] D. Ji, H. Feng, and W. Ge, "The existence of symmetric positive solutions for some nonlinear equation systems," Applied Mathematics and Computation, vol. 197, no. 1, pp. 51-59, 2008. 